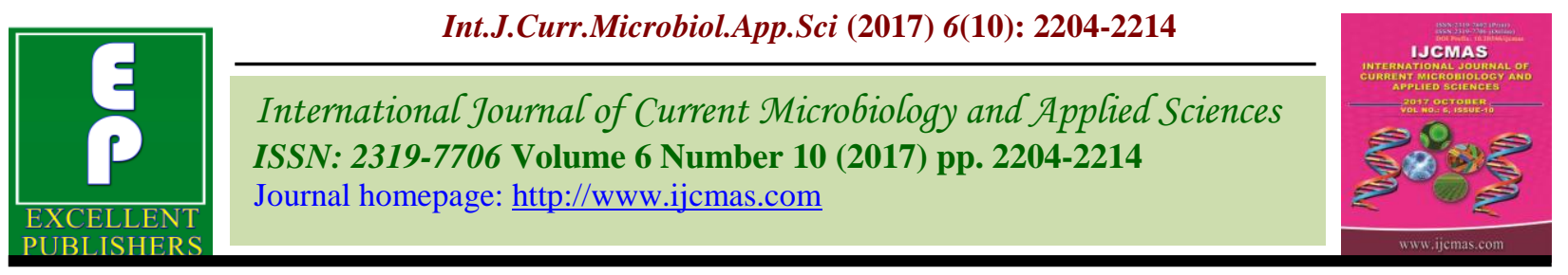

Original Research Article https://doi.org/10.20546/ijcmas.2017.610.261

\title{
Growth and Development of Spodopter litura (fab.) on Dual Toxin Transgenic Bt Cotton
}

\author{
Y.N. Venkatesh ${ }^{1 *}$, N.V.V.S.D. Prasad ${ }^{2}$, K.B. Ramesh $^{1}$, N. Srinivasa ${ }^{1}$ and \\ D.V. Sai Ram Kumar ${ }^{3}$ \\ ${ }^{1}$ Division of Entomology, ICAR-Indian Agricultural Research Institute, \\ New Delhi-110012, India \\ ${ }^{2}$ Regional Agricultural Research Station, ANGRAU, Lam, Guntur, AP-522034, India \\ ${ }^{3}$ ANGRAU, College of Agriculture, Bapatla, A.P- 522101, India \\ *Corresponding author
}

\section{A B S T R A C T}

Keywords

Cotton, Spodoptera litura, Boll guard II and Hybrids.

Article Info

Accepted:

21 September 2017

Available Online:

10 October 2017
The study was conducted to know the effect of dual toxin $B t$ cotton on Spodoptera litura larvae at regional agricultural research station, Lam, Guntur, A.P. during 2014-2015. The data revealed that survival rate of $3^{\text {rd }}$ instar larvae was higher and recorded only 30 - 35 per cent mortality, whereas the influence of BG II hybrids on grown up $4^{\text {th }}$ and $5^{\text {th }}$ instar larvae was very minimal no mortality was recorded and BG I and non $B t$ hybrids had shown no mortality. The mean weight of larva fed on BG II cotton leaves was found to be very low, whereas BG I hybrids shows marginal influence and non $B t$ versions did not affect the larval or pupal weight and weight gains were more or less similar.

\section{Introduction}

Advances made in the genetic engineering led to the development of first biotechnological tool in the world, the Bacillus thuringiensis (Bt) CrylAc gene (crystal protein delta endotoxin) incorporated cotton (Bollgard) which was commercially cultivated since 2002 in India. The first successful example of the biotechnology in crop protection is Bollgard. The Bollgard imparts insect resistance by virtue of which the cotton plant is able to resist attack of lepidopteron insect pests. This in-built protection in Bollgard cotton is provided by the presence of the $B t$ gene in the seed. Bollgard cultivars express the Cry1Ac protein and are active against larval stages of selected lepidopteron insects (Perlak et al., 1990). The technology has been anticipated to address bollworm infestation and significant reduction in the overall use of insecticides (Fernandez and McBride, 2000). $B t$ cotton technology has a capability of reducing insect pest infestations by 60-90 per cent under field conditions (Kranthi et al., 2004).

India is planning to produce 40 million bales of lint by $2050 \mathrm{AD}$ to meet the anticipated domestic and export requirements. To fulfill 
this projected requirement, the cotton production has to be increased by 15 per cent over existing levels which has to come mainly from increased productivity. Reasons for the low productivity of cotton in India are cultivation under rainfed conditions, predominance of pests and diseases, inadequate and unscientific method of cultivation.

It has been reported that 162 insect pests attack cotton in India (Lingappa, 2001). But key production constraints are sucking pests and bollworms are which cause losses to the tune of 30-80\% (Patil, 1998). Leaf worm, Spodoptera litura Fab. (Noctuidae: Lepidoptera) is one of the most destructive pests of cotton which feeds on foliage and sometimes bolls, is a secondary pest of cotton (Allen et al., 2000).

The three R's (Resistance, Resurgence and Residue) are the most potential problems associated with indiscriminate use of insecticides in cotton agro-ecosystem (Armes et al., 1992). Moreover, the modern production technologies which rely heavily on the use of chemicals have significantly lowered the cost benefit ratio in cotton.

It has been found that $S$. litura has a greater potential to survive in the presence of $B t$ toxins when compared to other bollworms. In the present study, two different plant parts namely leaves and squares of $B t$ and non $B t$ cotton hybrids were tested for their affect on third, fourth and fifth instar larvae of S. litura.

\section{Materials and Methods}

Growth and development of Spodoptera litura (Fab.) On Dual Toxin Transgenic Bt Cotton was conducted at Regional Agricultural Research Station, Lam, Guntur, A.P. during kharif, 2014-15. The two cotton hybrids viz., Mallika BG I and Jaadoo BG I containing
CrylAc, the two stacked $B t$ cotton hybrids viz., Mallika BG II and Jaadoo BG II containing Cry $1 \mathrm{Ac}+$ Cry $2 \mathrm{Ab}$ and their corresponding non $B t$ versions i.e., Mallika non $B t$ and Jaadoo non $B t$ were chosen for the research work. Seed material was obtained from M/S Kaveri seeds Pvt. Ltd and Nuzuveedu seeds Pvt. Ltd, Guntur.

A bulk plot of $19.44 \mathrm{~m}^{2}$ each of BG I (Cry1Ac), stacked Bt (Cry1Ac + Cry2Ab) (BG II) cotton hybrids of Mallika, Jaadoo and their corresponding non $B t$ hybrids were raised by following recommended agronomic practices with insecticidal protection to control sucking pests during the crop season. The experiment was conducted in CRD design in the lab with 6 treatments and 4 replications for each replication with 10 larvae.

Investigation was conducted in the laboratory on the leaves and squares of 70 to 80 days old crop with the third, fourth and fifth larval instars by feeding the leaves of $B t$ and stacked $B t$ cotton and their corresponding non $B t$ versions as control. Laboratory reared larvae were released separately on leaves and squares of 70 to 80 days old crop with four replications.

Comparative growth and development studies on S. litura larval instars were conducted for third, fourth and fifth larval instars by allowing them to feed on leaves and squares of different transgenic $B t$ cotton hybrids and non $B t$ cotton hybrids. Bioassay was conducted with BG I (Cry1Ac) and stacked Bt $($ Cry1Ac $+2 A b)$ (BG II) cotton hybrids of Mallika, Jaadoo and their corresponding non $B t$ versions against $S$. litura to study the per cent mortality, larval survival, pupal weight at 3, 7 and 11days interval of third, fourth and fifth larval instars and finally percent pupation and percent adult emergence was recorded. 


\section{Statistical analysis}

The larval survival and pupal weight was subjected to square root transformation and the variance was calculated. The data obtained on per cent mortality in bioassay was subjected to arcsin transformation and statistically analyzed by Completely Randomized Design (CRD). Means in simple CRD analysis were separated by Duncan's multiple range test (Duncan, 1951).

\section{Results and Discussion}

\section{Effect of test hybrids on larval mortality}

\section{Leaves}

\section{Third instar}

The mortality was nil on leaves of 70-80 days old crop of BG I and non $B t$ hybrids at 3, 7 and 11 days after release, while mortality was recorded on the stacked $B t$ (BG II) hybrids. The mortality at 3 days after release was 22.50 and 17.50 per cent with significant difference on Jaadoo BG II and Mallika BG II hybrids respectively.

The mortality was 15.00 per cent and 12.50 per cent on leaves of both the stacked $B t$ (BG II) hybrids at 7 days after release, while the total mortality was 35.00 and 32.50 per cent with significant difference when fed on leaves of Jaadoo BG II and Mallika BG II hybrids respectively at 11 days after release.

\section{Fourth and fifth instar}

The $4^{\text {th }}$ and $5^{\text {th }}$ instar larvae recorded no mortality on leaves of $70-80$ days old crop of Jaadoo BG II and Mallika BG II and their corresponding BG I, non $B t$ cotton hybrids at 3,7 , and 11 days after release. The stacked $B t$ (BG II) hybrids were found more resistant to fourth and fifth instars as no mortality was recorded on both the stacked Bt (BG II) hybrids at 3, 7 and 11 days after release. There is no significant difference between Jaadoo BG II and Mallika BG II cotton hybrids and their corresponding BG I and non- $B t$ hybrids (Table 1 ).

\section{Squares}

\section{Third instar}

The mortality was nil on squares of BG I and non $B t$ hybrids at 3, 7 and 11days after release, while mortality was recorded on the stacked $B t$ (BG II) hybrids. The mortality at 3 days after release was 12.50 per cent on both Jaadoo BG II and Mallika BG II hybrids. The mortality was 12.50 per cent on both the stacked Bt (BG II) hybrids at 7 days after release, while the total mortality was 25.00 per cent with significant difference when fed on squares of Jaadoo BG II and Mallika BG II and BG I and non $B t$ hybrids at 11 days after release (Table 2).

\section{Fourth and fifth instar}

No mortality on squares of Jaadoo BG II and Mallika BG II and their corresponding BG I, non $B t$ cotton hybrids at 3 and 7 days after release. The stacked $B t$ (BG II) hybrids were found more resistant to fourth and fifth instars as no mortality was recorded on both the stacked Bt (BG II) hybrids at 3 and 7 days after release. No significant difference between Jaadoo BG II and Mallika BG II cotton hybrids and their corresponding BG I and non- $B t$ hybrids at all the observations (Table 2).

\section{Effect of test hybrids on larval weight}

The mean weight of larvae that survived beyond 3 and 7 days after release on leaves and squares of 70-80 days old crop from all the six test hybrids was recorded for third, fourth and fifth instars and presented in table 3. 


\section{Leaves}

The minimum larval weight gain was recorded when fed on leaves of Jaadoo and Mallika BG II hybrids. Jaadoo and Mallika BG II hybrids with least increase in larval weight was significantly superior over other test hybrids. The mean larval weight of third instar larvae fed on leaves was minimum in Mallika (146.56 mg/larva) and Jaadoo (150.17 mg/larva) BGII hybrids at 3 days after feeding compared to other test hybrids. The maximum weight gained was on Mallika non- $B t(283.75 \mathrm{mg} /$ larva) followed by Jaadoo non-Bt (201.65 mg/larva), Mallika BG I (178.80 mg/larva) and Jaadoo BG I (175.38 mg/larva) (Table 3).

At 7 days after release maximum weight gain was on Mallika non-Bt (602.22 mg/larva) followed by Mallika BG I (559.25mg/larva), Jaadoo non Bt (529.50 mg/larva), Jaadoo BG I ( $528.87 \mathrm{mg} / \mathrm{larva})$ and minimum weight gain was in Jaadoo BG II (452.12 $\mathrm{mg} /$ larva) followed by Mallika BG II (470.12 mg/larva). It means Jaadoo BG II and Mallika BG II on a par and recorded lowest weight gain compared to other hybrids. The per cent weight gain after 7 days after feeding was maximum in Mallika non $B t(90.30 \%)$ followed by Mallika BG I (89.56 \%), Jaadoo non $B t(88.97 \%)$, Jaadoo BG I $(88.96 \%)$ and the per cent weight gain during third instar was lowest $(87.08 \%)$ in Jaadoo BG II, followed by $(87.58 \%)$ in Mallika BG II which were statistically superior over other hybrids.

The mean larval weight of fourth instar larvae after feeding for 3 days was maximum in Mallika non-Bt $(537.10 \mathrm{mg} / \mathrm{larva})$ followed by Jaadoo non- $B t(519.93 \mathrm{mg} / \mathrm{larva})$ Mallika BG I (480.00 mg/larva) and Jaadoo BG I (466.23 mg/larva) and minimum in Jaadoo BGII hybrid (389.23 mg/larva) followed by Mallika BG II (389.78mg/larva). After three days of feeding the per cent weight gain was low (53.73 \%) in Jaadoo BG II and Mallika BG II (53.79\%) compared to other test hybrids. The maximum weight gain was noticed in Mallika non-Bt (66.47\%) followed by Jaadoo non $B t(65.36 \%)$, Mallika BG I (62.48\%), and Jaadoo BG I (61.37\%) All these hybrids are statistically on par and significantly different from Jaadoo and Mallika BGII hybrids. The decreased mortality, lower larval and pupal weight growth of S. litura on Jaadoo and Mallika BGII observed in the present studies are in conformity with the results of Soujanya (2011), Naik, et al., (2013).

\section{Squares}

The mean larval weight of third instar larvae fed on leaves was minimum in Mallika (128.81 mg/larva) and Jaadoo (164.42 $\mathrm{mg} / \mathrm{larva}$ ) BGII hybrids at 3 days after feeding compared to other test hybrids. The maximum weight gain was on Mallika BG I (350.40 $\mathrm{mg} / \mathrm{larva}$ ) followed by Jaadoo BG I (297.00 $\mathrm{mg} / \mathrm{larva}$ ), Jaadoo non- $B t$ (268.95mg/larva) and Mallika non- $B t$ (239.80mg/larva) (Table 4).

At 7 days after release maximum weight gain was on Mallika non- $B t$ (565.73 mg/larva) followed by Jaadoo BG I $(502.88 \mathrm{mg} /$ larva), Jaadoo non- $B t$ ( $488.58 \mathrm{mg} /$ larva), Mallika BG I (451.33 mg/larva) and minimum weight gain was in Jaadoo BG II (314.55 mg/larva) followed by Mallika BG II ( $366.43 \mathrm{mg} / \mathrm{larva})$. It means Jaadoo BG II and Mallika BG II significantly differ in larval weight gain compared to other hybrids. The per cent weight gain after 7 days after feeding was maximum in Mallika non Bt $(84.90 \%)$ followed by Jaadoo BG I (83.08 \%), Jaadoo non $B t(82.52 \%)$, Mallika BG I (81.08\%) and the per cent weight gain during third instar was lowest $(72.85 \%)$ in Jaadoo BGII, followed by (76.69 \%) in Mallika BG II which are statistically superior over other hybrids. 
Table.1 Mortality of larval instars of S. litura on leaves of test hybrids

\begin{tabular}{|c|c|c|c|c|c|c|c|c|}
\hline \multirow{3}{*}{ Treatments } & \multicolumn{8}{|c|}{$\%$ mortality } \\
\hline & \multicolumn{3}{|c|}{ III instar } & \multirow{2}{*}{$\begin{array}{c}\text { Total } \\
\text { mortalit } \\
\mathbf{y}\end{array}$} & \multicolumn{2}{|c|}{ IV instar } & \multirow{2}{*}{$\begin{array}{c}\text { Total } \\
\text { mortalit } \\
\mathbf{y}\end{array}$} & \multirow[b]{2}{*}{$\mathrm{V}$ instar } \\
\hline & 3DAR & 7DAR & 11DAR & & 3DAR & 7DAR & & \\
\hline Jaadoo BG I & $\begin{array}{r}0.00 \\
(0.00)^{\mathrm{c}}\end{array}$ & $\begin{array}{c}0.00 \\
(0.00)^{\mathrm{b}}\end{array}$ & $\begin{array}{c}0.00 \\
(0.00)\end{array}$ & 0.00 & $\begin{array}{c}0.00 \\
(0.00)\end{array}$ & $\begin{array}{c}0.00 \\
(0.00)\end{array}$ & 0.00 & 0.00 \\
\hline Mallika BG I & $\begin{array}{c}0.00 \\
(0.00)^{\mathrm{c}}\end{array}$ & $\begin{array}{c}0.00 \\
(0.00)^{\mathrm{b}}\end{array}$ & $\begin{array}{c}0.00 \\
(0.00)\end{array}$ & 0.00 & $\begin{array}{c}0.00 \\
(0.00)\end{array}$ & $\begin{array}{c}0.00 \\
(0.00)\end{array}$ & 0.00 & 0.00 \\
\hline Jaadoo BG II & $\begin{array}{c}22.50 \\
(28.28)^{\mathrm{a}}\end{array}$ & $\begin{array}{c}12.50 \\
(20.46)^{\mathrm{a}}\end{array}$ & $\begin{array}{c}0.00 \\
(0.00)\end{array}$ & 35.00 & $\begin{array}{c}0.00 \\
(0.00)\end{array}$ & $\begin{array}{c}0.00 \\
(0.00)\end{array}$ & 0.00 & 0.00 \\
\hline Mallika BG II & $\begin{array}{c}17.50 \\
(24.67)^{b} \\
\end{array}$ & $\begin{array}{c}15.00 \\
(22.50)^{\mathrm{a}} \\
\end{array}$ & $\begin{array}{c}0.00 \\
(0.00) \\
\end{array}$ & 32.50 & $\begin{array}{c}0.00 \\
(0.00) \\
\end{array}$ & $\begin{array}{c}0.00 \\
(0.00) \\
\end{array}$ & 0.00 & 0.00 \\
\hline Jaadoo non- $B t$ & $\begin{array}{c}0.00 \\
(0.00)^{\mathrm{c}}\end{array}$ & $\begin{array}{c}0.00 \\
(0.00)^{\mathrm{b}}\end{array}$ & $\begin{array}{c}0.00 \\
(0.00)\end{array}$ & 0.00 & $\begin{array}{c}0.00 \\
(0.00)\end{array}$ & $\begin{array}{c}0.00 \\
(0.00)\end{array}$ & 0.00 & 0.00 \\
\hline Mallika non- $B t$ & $\begin{array}{c}0.00 \\
(0.00)^{\mathrm{c}}\end{array}$ & $\begin{array}{c}0.00 \\
(0.00)^{\mathrm{b}}\end{array}$ & $\begin{array}{c}0.00 \\
(0.00)\end{array}$ & 0.00 & $\begin{array}{c}0.00 \\
(0.00)\end{array}$ & $\begin{array}{c}0.00 \\
(0.00)\end{array}$ & 0.00 & 0.00 \\
\hline F-Test & Sig & Sig & NS & & NS & NS & & NS \\
\hline $\mathrm{SEM} \pm$ & 0.6 & 1.27 & - & & - & - & & - \\
\hline $\mathrm{CD}(\mathrm{P}=0.05)$ & 1.79 & 3.77 & - & & - & - & & - \\
\hline
\end{tabular}

Figures in parentheses are angular transformed values

Numbers followed by same superscript are not statistically different $(\mathrm{P}=0.05)$

Table.2 Mortality of larval instars of S. litura on squares of test hybrids

\begin{tabular}{|c|c|c|c|c|c|c|c|c|}
\hline \multirow{3}{*}{ Treatments } & \multicolumn{8}{|c|}{$\%$ mortality } \\
\hline & \multicolumn{3}{|c|}{ III instar } & \multirow{2}{*}{$\begin{array}{c}\text { Total } \\
\text { mortali } \\
\text { ty }\end{array}$} & \multicolumn{2}{|c|}{ IV instar } & \multirow{2}{*}{$\begin{array}{c}\text { Total } \\
\text { mortality }\end{array}$} & \multirow{2}{*}{$\mathrm{V}$ instar } \\
\hline & 3DAR & 7DAR & 11DAR & & 3DAR & 7DAR & & \\
\hline Jaadoo BG I & $\begin{array}{c}0.00 \\
(0.00)^{\mathrm{b}}\end{array}$ & $\begin{array}{c}0.00 \\
(0.00)^{\mathrm{b}}\end{array}$ & $\begin{array}{c}0.00 \\
(0.00) \\
\end{array}$ & 0.00 & $\begin{array}{c}0.00 \\
(0.00) \\
\end{array}$ & $\begin{array}{c}0.00 \\
(0.00) \\
\end{array}$ & 0.00 & 0.00 \\
\hline Mallika BG I & $\begin{array}{c}0.00 \\
(0.00)^{\mathrm{b}}\end{array}$ & $\begin{array}{c}0.00 \\
(0.00)^{\mathrm{b}}\end{array}$ & $\begin{array}{c}0.00 \\
(0.00)\end{array}$ & 0.00 & $\begin{array}{c}0.00 \\
(0.00)\end{array}$ & $\begin{array}{c}0.00 \\
(0.00)\end{array}$ & 0.00 & 0.00 \\
\hline Jaadoo BG II & $\begin{array}{c}12.50 \\
(20.61)^{\mathrm{a}}\end{array}$ & $\begin{array}{c}12.50 \\
(20.46)^{\mathrm{a}}\end{array}$ & $\begin{array}{c}0.00 \\
(0.00) \\
\end{array}$ & 25.00 & $\begin{array}{c}0.00 \\
(0.00) \\
\end{array}$ & $\begin{array}{c}0.00 \\
(0.00) \\
\end{array}$ & 0.00 & 0.00 \\
\hline Mallika BG II & $\begin{array}{c}12.50 \\
(20.46)^{\mathrm{a}} \\
\end{array}$ & $\begin{array}{c}12.50 \\
(20.46)^{\mathrm{a}} \\
\end{array}$ & $\begin{array}{c}0.00 \\
(0.00) \\
\end{array}$ & 25.00 & $\begin{array}{c}0.00 \\
(0.00) \\
\end{array}$ & $\begin{array}{c}0.00 \\
(0.00) \\
\end{array}$ & 0.00 & 0.00 \\
\hline Jaadoo non- $B t$ & $\begin{array}{c}0.00 \\
(0.00)^{\mathrm{b}}\end{array}$ & $\begin{array}{c}0.00 \\
(0.00)^{\mathrm{b}}\end{array}$ & $\begin{array}{c}0.00 \\
(0.00) \\
\end{array}$ & 0.00 & $\begin{array}{c}0.00 \\
(0.00) \\
\end{array}$ & $\begin{array}{c}0.00 \\
(0.00) \\
\end{array}$ & 0.00 & 0.00 \\
\hline Mallika non- $B t$ & $\begin{array}{c}0.00 \\
(0.00)^{\mathrm{b}}\end{array}$ & $\begin{array}{c}0.00 \\
(0.00)^{\mathrm{b}}\end{array}$ & $\begin{array}{c}0.00 \\
(0.00)\end{array}$ & 0.00 & $\begin{array}{c}0.00 \\
(0.00)\end{array}$ & $\begin{array}{c}0.00 \\
(0.00)\end{array}$ & 0.00 & 0.00 \\
\hline F-Test & Sig & Sig & NS & & NS & NS & & NS \\
\hline $\mathrm{SEm} \pm$ & 0.98 & 1.17 & & & - & - & & - \\
\hline $\mathrm{CD}(\mathrm{P}=0.05)$ & 2.90 & 3.49 & & & - & - & & - \\
\hline
\end{tabular}

Figures in parentheses are angular transformed values

Numbers followed by same superscript are not statistically different $(\mathrm{P}=0.05)$ 
Table.3 Effect of test hybrids leaves on S. litura larval weight

\begin{tabular}{|c|c|c|c|c|c|}
\hline \multirow[t]{2}{*}{ Treatments } & \multicolumn{2}{|c|}{$\begin{array}{c}\text { Mean weight (mg/larva) of III } \\
\text { instar after }\end{array}$} & \multirow{2}{*}{$\begin{array}{l}\text { Weight gain } \\
(\%)\end{array}$} & \multirow{2}{*}{$\begin{array}{c}\begin{array}{c}\text { Mean weight } \\
\text { (mg/larva) of IV } \\
\text { instar after }\end{array} \\
\text { 3 DAR }\end{array}$} & \multirow{2}{*}{$\begin{array}{l}\text { Weight gain } \\
(\%)\end{array}$} \\
\hline & 3 DAR & 7 DAR & & & \\
\hline Jaadoo BG I & $\begin{array}{c}175.38 \\
(13.25)^{\mathrm{bc}}\end{array}$ & $\begin{array}{l}528.87 \\
(23.02)^{\mathrm{b}}\end{array}$ & 88.96 & $\begin{array}{c}466.23 \\
(21.59)^{\mathrm{ab}}\end{array}$ & 61.37 \\
\hline Mallika BG I & $\begin{array}{l}178.80 \\
(13.39)^{\mathrm{bc}}\end{array}$ & $\begin{array}{c}559.25 \\
(23.66)^{\mathrm{ab}}\end{array}$ & 89.56 & $\begin{array}{l}480.00 \\
(21.93)^{\mathrm{a}}\end{array}$ & 62.48 \\
\hline Jaadoo BG II & $\begin{array}{l}150.17 \\
(12.23)^{\mathrm{c}}\end{array}$ & $\begin{array}{l}452.12 \\
(21.27)^{\mathrm{c}}\end{array}$ & 87.08 & $\begin{array}{l}389.23 \\
(19.70)^{\mathrm{b}}\end{array}$ & 53.73 \\
\hline Mallika BG II & $\begin{array}{l}146.56 \\
(12.11)^{\mathrm{c}}\end{array}$ & $\begin{array}{l}470.12 \\
(21.70)^{\mathrm{c}}\end{array}$ & 87.58 & $\begin{array}{c}389.78 \\
(19.69)^{\mathrm{b}}\end{array}$ & 53.79 \\
\hline Jaadoo non- $B t$ & $\begin{array}{l}201.65 \\
(14.23)^{\mathrm{b}}\end{array}$ & $\begin{array}{l}529.50 \\
(23.03)^{\mathrm{b}}\end{array}$ & 88.97 & $\begin{array}{l}519.93 \\
(22.82)^{\mathrm{a}}\end{array}$ & 65.36 \\
\hline Mallika non- $B t$ & $\begin{array}{l}283.75 \\
(16.79)^{\mathrm{a}}\end{array}$ & $\begin{array}{l}602.22 \\
(24.54)^{\mathrm{a}}\end{array}$ & 90.30 & $\begin{array}{l}537.10 \\
(23.17)^{\mathrm{a}}\end{array}$ & 66.47 \\
\hline F- Test & Sig & Sig & & Sig & \\
\hline $\mathrm{SEm} \pm$ & 0.61 & 0.42 & & 0.65 & \\
\hline $\mathrm{CD}(\mathrm{P}=0.05)$ & 1.80 & 1.25 & & 1.93 & \\
\hline Initial weight & \multicolumn{2}{|c|}{58.4} & & 180.1 & \\
\hline
\end{tabular}

Figures in parentheses are square root transformed values

Numbers followed by same superscript are not statistically different $(\mathrm{P}=0.05)$

Table.4 Effect of test hybrid squares on S. litura larval weight

\begin{tabular}{|c|c|c|c|c|c|}
\hline \multirow[t]{2}{*}{ Treatments } & \multicolumn{2}{|c|}{$\begin{array}{c}\text { Mean weight (mg/larva) of III } \\
\text { instar after }\end{array}$} & \multirow{2}{*}{$\begin{array}{c}\text { Weight } \\
\text { gain } \\
(\%)\end{array}$} & \multirow{2}{*}{$\begin{array}{c}\begin{array}{c}\text { Mean weight of } \\
\text { (mg/larva) of IV instar } \\
\text { after }\end{array} \\
\text { 3 DAR }\end{array}$} & \multirow{2}{*}{$\begin{array}{c}\text { Weigh } \\
\text { gain } \\
(\%)\end{array}$} \\
\hline & 3 DAR & 7 DAR & & & \\
\hline Jaadoo BG I & $\begin{array}{c}297.00 \\
(17.25)^{\mathrm{ab}}\end{array}$ & $\begin{array}{l}502.88 \\
(22.44)^{\mathrm{b}}\end{array}$ & 83.02 & $\begin{array}{l}486.93 \\
(22.09)^{\mathrm{a}} \\
\end{array}$ & 65.44 \\
\hline Mallika BG I & $\begin{array}{c}350.40 \\
(18.74)^{\mathrm{a}}\end{array}$ & $\begin{array}{l}451.33 \\
(21.22)^{\mathrm{c}}\end{array}$ & 81.08 & $\begin{array}{l}480.00 \\
(21.93)^{\mathrm{a}}\end{array}$ & 64.94 \\
\hline Jaadoo BG II & $\begin{array}{l}164.42 \\
(12.57)^{\mathrm{c}}\end{array}$ & $\begin{array}{l}314.55 \\
(17.76)^{\mathrm{e}} \\
\end{array}$ & 72.85 & $\begin{array}{c}389.23 \\
(19.70)^{\mathrm{b}}\end{array}$ & 56.76 \\
\hline Mallika BG II & $\begin{array}{l}128.81 \\
(11.37)^{\mathrm{c}}\end{array}$ & $\begin{array}{c}366.43 \\
(19.16)^{\mathrm{d}}\end{array}$ & 76.69 & $\begin{array}{c}339.78 \\
(18.43)^{\mathrm{b}}\end{array}$ & 50.47 \\
\hline Jaadoo non- $B t$ & $\begin{array}{c}268.95 \\
(16.34)^{\mathrm{ab}} \\
\end{array}$ & $\begin{array}{c}488.58 \\
(22.12)^{\mathrm{bc}} \\
\end{array}$ & 82.52 & $\begin{array}{c}527.43 \\
(22.98)^{\mathrm{a}} \\
\end{array}$ & 68.09 \\
\hline Mallika non- $B t$ & $\begin{array}{c}239.80 \\
(15.51)^{\mathrm{b}} \\
\end{array}$ & $\begin{array}{l}565.73 \\
(23.80)^{\mathrm{a}} \\
\end{array}$ & 84.90 & $\begin{array}{c}537.10 \\
(23.17)^{\mathrm{a}} \\
\end{array}$ & 68.67 \\
\hline F- Test & Sig & Sig & & Sig & \\
\hline SEm \pm & 0.81 & 0.41 & & 0.51 & \\
\hline $\mathrm{CD}(\mathrm{P}=0.05)$ & 2.40 & 1.21 & & 1.51 & \\
\hline Initial weight & \multicolumn{2}{|c|}{85.4} & & 168.3 & \\
\hline
\end{tabular}

Figures in parentheses are square root transformed values

Numbers followed by same superscript are not statistically different $(\mathrm{P}=0.05)$ 
Table.5 Per cent pupation of surviving larvae of S. litura on test hybrids

\begin{tabular}{|c|c|c|c|c|c|c|}
\hline \multirow{2}{*}{ Treatments } & \multicolumn{7}{|c|}{ Pupation (\%) } \\
\cline { 2 - 7 } & \multicolumn{3}{|c|}{ Leaves } & \multicolumn{3}{c|}{ Squares } \\
\cline { 2 - 7 } & III instar & IV instar & V instar & III instar & IV instar & V instar \\
\hline \multirow{2}{*}{ Jaadoo BG I } & 100.00 & 100.00 & 100.00 & 100.00 & 100 & 100 \\
& $(90.00)^{\mathrm{a}}$ & $(90.00)$ & $(90.00)$ & $(90.00)^{\mathrm{a}}$ & $(90.00)$ & $(90.00)$ \\
\hline \multirow{2}{*}{ Mallika BG I } & 100.00 & 100.00 & 100.00 & 100.00 & 100 & 100 \\
& $(90.00)^{\mathrm{a}}$ & $(90.00)$ & $(90.00)$ & $(90.00)^{\mathrm{a}}$ & $(90.00)$ & $(90.00)$ \\
\hline \multirow{2}{*}{ Jaadoo BG II } & 65.00 & 100.00 & 100.00 & 75.00 & 100 & 100 \\
& $(53.77)^{\mathrm{b}}$ & $(90.00)$ & $(90.00)$ & $(60.11)^{\mathrm{b}}$ & $(90.00)$ & $(90.00)$ \\
\hline \multirow{2}{*}{ Mallika BG II } & 67.50 & 100.00 & 100.00 & 75.00 & 100 & 100 \\
& $(55.28)^{\mathrm{b}}$ & $(90.00)$ & $(90.00)$ & $(60.63)^{\mathrm{b}}$ & $(90.00)$ & $(90.00)$ \\
\hline \multirow{2}{*}{ Jaadoo non- Bt } & 100.00 & 100.00 & 100.00 & 100 & 100 & 100 \\
& $(90.00)^{\mathrm{a}}$ & $(90.00)$ & $(90.00)$ & $(90.00)^{\mathrm{a}}$ & $(90.00)$ & $(90.00)$ \\
\hline \multirow{2}{*}{ Mallika non- Bt } & 100.00 & 100.00 & 100.00 & 100 & 100 & 100 \\
& $(90.00)^{\mathrm{a}}$ & $(90.00)$ & $(90.00)$ & $(90.00)^{\mathrm{a}}$ & $(90.00)$ & $(90.00)$ \\
\hline F-test & Sig & & & Sig & NS & NS \\
\hline SEm \pm & 0.94 & - & - & 1.98 & - & - \\
\hline CD (P=0.05) & 2.40 & - & - & 5.90 & - & - \\
\hline
\end{tabular}

Figures in parentheses are angular transformed values

Numbers followed by same superscript are not statistically different $(\mathrm{P}=0.05)$

Table.6 Effect of test hybrids on pupal weight of S. litura

\begin{tabular}{|c|c|c|c|c|c|c|}
\hline \multirow{3}{*}{ Treatments } & \multicolumn{6}{|c|}{ Pupal weight (mg/pupa) } \\
\hline & \multicolumn{3}{|c|}{ Leaves } & \multicolumn{3}{|c|}{ Squares } \\
\hline & III instar & IV instar & V instar & III instar & IV instar & $\mathrm{V}$ instar \\
\hline Jaadoo BG I & $\begin{array}{c}206.53 \\
(14.40)^{\mathrm{a}}\end{array}$ & $\begin{array}{c}281.75 \\
(16.81)^{\mathrm{a}}\end{array}$ & $\begin{array}{c}256.58 \\
(16.02)^{\mathrm{a}}\end{array}$ & $\begin{array}{c}160.83 \\
(12.70)^{\mathrm{a}}\end{array}$ & $\begin{array}{c}244.78 \\
(15.65)^{b}\end{array}$ & $\begin{array}{c}268.25 \\
(16.40)^{\mathrm{a}}\end{array}$ \\
\hline Mallika BG I & $\begin{array}{c}210.55 \\
(14.54)^{\mathrm{a}}\end{array}$ & $\begin{array}{c}267.57 \\
(16.37)^{\mathrm{a}} \\
\end{array}$ & $\begin{array}{c}242.75 \\
(15.60)^{\mathrm{ab}}\end{array}$ & $\begin{array}{c}152.13 \\
(12.37)^{\mathrm{a}}\end{array}$ & $\begin{array}{c}281.58 \\
(16.80)^{\mathrm{a}}\end{array}$ & $\begin{array}{c}260.23 \\
(16.15)^{\mathrm{a}}\end{array}$ \\
\hline Jaadoo BG II & $\begin{array}{c}167.27 \\
(12.93)^{\mathrm{b}} \\
\end{array}$ & $\begin{array}{c}223.37 \\
(14.97)^{\mathrm{b}} \\
\end{array}$ & $\begin{array}{c}210.90 \\
(14.55)^{\mathrm{c}} \\
\end{array}$ & $\begin{array}{c}117.83 \\
(10.89)^{\mathrm{b}} \\
\end{array}$ & $\begin{array}{c}208.53 \\
(14.46)^{\mathrm{c}} \\
\end{array}$ & $\begin{array}{c}243.58 \\
(15.63)^{\mathrm{ab}} \\
\end{array}$ \\
\hline Mallika BG II & $\begin{array}{c}166.75 \\
(12.95)^{\mathrm{b}}\end{array}$ & $\begin{array}{c}213.80 \\
(14.65)^{\mathrm{b}}\end{array}$ & $\begin{array}{c}220.43 \\
(14.87)^{\mathrm{bc}}\end{array}$ & $\begin{array}{c}117.66 \\
(10.88)^{\mathrm{b}}\end{array}$ & $\begin{array}{c}202.95 \\
(14.27)^{\mathrm{c}} \\
\end{array}$ & $\begin{array}{c}223.1 \\
(15.07)^{\mathrm{b}}\end{array}$ \\
\hline Jaadoo non- $B t$ & $\begin{array}{c}224.13 \\
(14.99)^{\mathrm{a}}\end{array}$ & $\begin{array}{c}278.20 \\
(16.70)^{\mathrm{a}}\end{array}$ & $\begin{array}{c}261.05 \\
(16.18)^{\mathrm{a}}\end{array}$ & $\begin{array}{c}166.73 \\
(12.94)^{\mathrm{a}}\end{array}$ & $\begin{array}{c}249.3 \\
(15.80)^{\mathrm{ab}}\end{array}$ & $\begin{array}{c}260.6 \\
(16.17)^{\mathrm{a}}\end{array}$ \\
\hline Mallika non- $B t$ & $\begin{array}{c}220.90 \\
(14.89)^{\mathrm{a}} \\
\end{array}$ & $\begin{array}{c}271.75 \\
(16.51)^{\mathrm{a}} \\
\end{array}$ & $\begin{array}{c}268.25 \\
(16.39)^{\mathrm{a}} \\
\end{array}$ & $\begin{array}{c}165.38 \\
(12.89)^{\mathrm{a}} \\
\end{array}$ & $\begin{array}{c}265.88 \\
(16.32)^{\mathrm{ab}} \\
\end{array}$ & $\begin{array}{c}268.75 \\
(16.40)^{\mathrm{a}} \\
\end{array}$ \\
\hline F-test & Sig & Sig & Sig & Sig & Sig & Sig \\
\hline SEm \pm & 0.43 & 0.26 & 0.3 & 0.45 & 0.34 & 0.28 \\
\hline $\mathrm{CD}(\mathrm{P}=0.05)$ & 1.27 & 0.77 & 0.9 & 1.35 & 1.02 & 0.82 \\
\hline
\end{tabular}

Figures in parentheses are square root transformed values

Numbers followed by same superscript are not statistically different $(\mathrm{P}=0.05)$ 
Table.7 Per cent adult emergence of S. litura on test hybrids

\begin{tabular}{|c|c|c|c|c|c|c|}
\hline \multirow{2}{*}{ Treatments } & \multicolumn{7}{|c|}{ Adult emergence (\%) } \\
\cline { 2 - 7 } & \multicolumn{3}{|c|}{ Leaves } & \multicolumn{3}{c|}{ Squares } \\
\cline { 2 - 7 } & III instar & IV instar & V instar & III instar & IV instar & V instar \\
\hline \multirow{2}{*}{ Jaadoo BG I } & 100.00 & 100.00 & 100.00 & 100.00 & 100.00 & 100.00 \\
& $(90.00)^{\mathrm{a}}$ & $(90.00)$ & $(90.00)$ & $(90.00)^{\mathrm{a}}$ & $(90.00)$ & $(90.00)$ \\
\hline \multirow{2}{*}{ Mallika BG I } & 100.00 & 100.00 & 100.00 & 100.00 & 100.00 & 100.00 \\
& $(90.00)^{\mathrm{a}}$ & $(90.00)$ & $(90.00)$ & $(90.00)^{\mathrm{a}}$ & $(90.00)$ & $(90.00)$ \\
\hline \multirow{2}{*}{ Jaadoo BG II } & 62.50 & 100.00 & 100.00 & 70.00 & 100.00 & 100.00 \\
& $(52.33)^{\mathrm{b}}$ & $(90.00)$ & $(90.00)$ & $(56.94)^{\mathrm{b}}$ & $(90.00)$ & $(90.00)$ \\
\hline \multirow{2}{*}{ Mallika BG II } & 65.00 & 100.00 & 100.00 & 70.00 & 100.00 & 100.00 \\
& $(53.99)^{\mathrm{b}}$ & $(90.00)$ & $(90.00)$ & $(56.94)^{\mathrm{b}}$ & $(90.00)$ & $(90.00)$ \\
\hline \multirow{2}{*}{ Jaadoo non- Bt } & 100.00 & 100.00 & 100.00 & 100.00 & 100.00 & 100.00 \\
& $(90.00)^{\mathrm{a}}$ & $(90.00)$ & $(90.00)$ & $(90.00)^{\mathrm{a}}$ & $(90.00)$ & $(90.00)$ \\
\hline \multirow{2}{*}{ Mallika non- Bt } & 100.00 & 100.00 & 100.00 & 100.00 & 100.00 & 100.00 \\
& $(90.00)^{\mathrm{a}}$ & $(90.00)$ & $(90.00)$ & $(90.00)^{\mathrm{a}}$ & $(90.00)$ & $(90.00)$ \\
\hline F-test & Sig & NS & NS & Sig & NS & NS \\
\hline SEm \pm & 1.99 & - & - & 1.49 & - & - \\
\hline CD $(\mathrm{P}=0.05)$ & 5.9 & - & - & 3.78 & - & - \\
\hline
\end{tabular}

Figures in parentheses are angular transformed values

Numbers followed by same superscript are not statistically different $(\mathrm{P}=0.05)$

The mean larval weight of fourth instar larvae after feeding for 3 days was maximum in Mallika non- $B t(537.10 \mathrm{mg})$ followed by Jaadoo non-Bt (527.43 mg) Jaadoo BG I (486.93 mg) and Mallika BG I (480.00 mg) and minimum in Mallika BG II hybrid (339.78 $\mathrm{mg}$ ) followed by Jaadoo BG II (389.23). Both staked $B t$ cotton hybrids are significantly different from other hybrids. After three days of feeding the per cent weight gain was low $(50.47 \%)$ in Mallika BG II and Jaadoo BG II (56.76\%) compared to other test hybrids. The maximum weight gain was noticed in Mallika non-Bt $(68.67 \%)$ followed by Jaadoo non $B t(68.09 \%)$, Jaadoo BG I (65.44\%), and Mallika BG I (64.94\%), All these hybrids are statistically on par and significantly different from Jaadoo and Mallika BG II hybrids.

\section{Effect of test hybrids on pupation}

The per cent pupation of surviving larvae of S. litura on leaves and squares of different test cotton hybrids (Table 5).

\section{Leaves}

There was $67.50 \%$ pupation in third instar in Mallika BG II and $65.00 \%$ pupation in Jaadoo BG II cotton hybrids and $100.00 \%$ in fourth and fifth instar larvae when fed on Jaadoo and Mallika BG II hybrids and this is significantly superior over other hybrids.

In fourth and fifth instars larvae, there was no mortality, hence all the larvae pupated at the end on all the hybrids.

In Jaadoo and Mallika BG II hybrids also $100.00 \%$ pupation was recorded in fourth and fifth instar larvae.

\section{Squares}

The per cent pupation of third instar larvae fed on squares of Jaadoo BG II and Mallika BG II was $75.00 \%$ and it was $100.00 \%$ in all the hybrids in third, fourth and fifth instars (Table 5). 


\section{Effect of test hybrids on pupal weight}

\section{Leaves}

The third instar larvae fed on leaves of Jaadoo non $B t$ recorded $224.13 \mathrm{mg} / \mathrm{pupa}$ weight followed by Mallika non-Bt (220.90 mg/pupa), Mallika BG I (210.55 mg/pupa) and Jaadoo BG I (206.53 mg/pupa) hybrids and all are statistically on par with each other except Jaadoo and Mallika BG II hybrids with only (167.27 and $166.75 \mathrm{mg} / \mathrm{pupa})$ of mean pupal weight respectively.

The maximum pupal weight of 281.75 $\mathrm{mg} / \mathrm{pupa}$ was recorded, when fourth instars larvae were fed on leaves of Jaadoo BG I hybrid and it is statistically on par with other hybrids except Jaadoo and Mallika BG II hybrids (Table 6). The fifth instar larvae fed on leaves of Mallika non $B t$ recorded 268.25 $\mathrm{mg} / \mathrm{pupa}$ of pupal weight followed by Jaadoo non-Bt (261.05 mg/pupa), Jaadoo BG I (256.58 mg/pupa) and Mallika BG I (242.75 $\mathrm{mg} / \mathrm{pupa}$ ) hybrids and all are statistically on par with each other except Jaadoo and Mallika BG II hybrid with only 210.90, $220.43 \mathrm{mg} / \mathrm{pupa}$ of mean pupal weight respectively. BG II cotton hybrids were statistically significant over the BG I and non$B t$ hybrids.

\section{Squares}

The mean pupal weight has increased gradually with increase in age of larvae on Jaadoo and Mallika BGII hybrids. The maximum pupal weight $(268.75 \mathrm{mg} /$ pupa $)$ was recorded on Mallika non $B t$ hybrid and it was statistically on par with Jaadoo BG I (268.25 mg/pupa), Mallika BG I (260.23 $\mathrm{mg} / \mathrm{pupa})$, Jaadoo non- $B t$ (260.6 mg/pupa) whereas Jaadoo BG II (243.58 mg/pupa) and Mallika BGII hybrid recorded lowest (223.10 $\mathrm{mg} /$ pupa) pupal weight when fifth instars larvae were reared on Mallika BG II hybrid.

\section{Effect of different test hybrids on adult emergence}

\section{Leaves}

Third instar larvae recorded 62.50, 65.00\% adult emergence in Jaadoo and Mallika BG II hybrids respectively, In fourth and fifth instar there was 100 per cent adult emergence in all the test hybrids (Table 7).

\section{Squares}

Third instar $70 \%$ adult emergence was recorded on both Jaadoo and Mallika BG II hybrids. Where as in other hybrids $100.00 \%$ adult emergence was recorded and all these are on par. Adult emergence was $100 \%$ for fourth and fifth instars reared on the all test hybrids (Table 7).

The results from the present investigation also clearly suggest that the BG II cotton hybrids which contains Cry1Ac protein, offer marginal influence on only third instar larvae, these findings are in close association with the results of Zeng et al., (1998) who reported that the development of S. exigua on BG I cotton was normal. The BG I cotton hybrids which were efficient against $H$. armigera as revealed from the references of Kranthi et al., (2005) and Seshamahalakshmi (2007) have not offered any efficacy against $S$. litura during the 70-80 days old crop.

The duration of mortality of S. litura on leaves of all the test hybrids was studied for all the larval instars during 70 to 80 days old crop, pertaining to $3^{\text {rd }}$ instar larvae, there was 35.00 per cent mortality of larvae at 7 days after release. The findings clearly suggest that BG II cotton hybrids have marginal mortality on third instar larvae only and The mortality of $4^{\text {th }}$ and $5^{\text {th }}$ instar larvae were zero which was non economical in pest management of cotton ecosystem. The BG I and non Bt cotton 
hybrids did not effect any larval instar at 7080 days crop. Hence, it was evident from the results that BG I cotton hybrids which were resistant and showed higher mortality to $H$. armigera (Vennila et al., 2006; Jeyakumar et al., 2007; Basavaraja et al., 2008) cannot offer any protection to $S$. litura.

It was clear from the results that larval or pupal weights of third, fourth and fifth larval instars were maximum when fed on leaves of non $B t$ and BG I cotton hybrids. The present findings derive support from Sparks and Norman (2002) and Basavaraja et al., (2008) who reported that larval weights were significantly higher on normal cotton leaves than transgenic $B t$ cotton leaves. The results from the data also revealed that the larvae survived after exposure to stacked $B t$ (BG II) leaves weighed less and developed into smaller pupae than the larvae fed on BG I and non $B t$ hybrids. The present findings are in conformity with Camberos et al., (2000), Donglin et al., (2006), Fitt et al., (2000) and Seshamahalakshmi (2007) who reported significant reduction in the larval and pupal weights when fed with stacked $B t$ (BG II) cotton leaves.

\section{References}

Allen, C.T., Kharboutli, M.S., Capps, C and Earnest, L.D. 2000. Effectiveness of Bollgard II cotton varieties against foliage and fruit feeding caterpillars in Arkansas. Proceedings Beltwide Cotton Conference. San Antonio, USA, 4-8 January. 2: 1093-1094.

Armes, N.S., Jadhav, R.D., Boud, G.S and King, A.B.S. 1992. Insecticide in Helicoverpa armigera in south India. Pesticide Sciences. 34: 355-366.

Basavaraja, H., Chhillar, B.S and Singh, R. 2008. Impact of transgenic Bt cotton on biological parameters of Spodoptera litura (Fab.) (Lepidoptera: Noctuidae).
Journal of Entomological research. 32(3): 183-186.

Camberos, U.N., Frias, I., Dugger, P and Richter, D. 2000. Development, survival and fecundity of the beet armyworm on $B t$ and non $B t$ cotton. Proceedings Beltwide Cotton Conference, San Antonio, USA, 4-8 January.2: 1337-1339.

Donglin, H., Qin, L.H and Xia, J.S. 2006. Effects of Zhongmiansuo 45 and Zhongmiansuo 41 on experimental population of Spodoptera litura. Acta Phytophylacica Sinica. 33(1): 1-5.

Duncan, D.B., 1951. A significance test for differences between ranked treatment means in an analysis of variance. Virginia Journal of Science. 2: 171-189.

Fernandez, C.J., and McBride, W.D. 2000. Genetically engineered crops for pest management in U.S. Agriculture. Agriculture Economic Report. Department of Agriculture, U.S.

Fitt, G.P., Mares, C.L., Baker, G., Mahon, R and Olsen, K. 2000. Influence of weather on population dynamics of Spodoptera a polyphagous pest. Journal of Ecobiology. 16(4): 267-274.

Jeyakumar, P., Tanwar, R.K., Jat, M.C., Dhandapani, A., Bambawale, O.M and Monga, D. 2007. Spodoptera litura: An emerging pest on $B t$ cotton (Cry1Ac) under North Indian conditions. Pesticide Research Journal. 19(2): 197-200.

Kranthi, K.R., Kranthi, S., Naidu, S., Dhawad, C.S., Mate, K., Wadaskar, R.M., Choudhary, R., Bharose, A., Siddhabatti, P and Patil, E. 2004. IRM and $B t$ cotton In: International Symposium on strategies for sustainable cotton production. Crop Protection. 2325: $15-32$.

Kranthi, K.R., Naidu, S., Dhawad, C.S., Tatwawadi, A., Mate, K., Patil, E., Bharose, A.A., Behere, G.T., Wadaskar, R.M and Kranthi, S. 2005. Temporal 
and intra-plant variability of Cry1Ac expression in $B t$ cotton and its influence on the survival of the cotton bollworm, Helicoverpa armigera (Hubner) (Noctuidae: Lepidoptera). Current Science. 89(2):291-298.

Lingappa, S., 2001. Tools for management of Helicoverpa in cotton. In: National seminar on sustainable cotton production to meet the future requirement of industry. Directorate of cotton development, Mumbai from oct 3-4: 75-85.

Naik, V.C.B., 2009. Spatial-Temporal distribution of $B t$ insecticidal protein in different $B t$ events and their efficacy against bollworms under field and laboratory conditions. Ph.D Thesis. Acharya N.G Ranga Agricultural University, Hyderabad.

Patil, B.V., 1998. Development IPM schedule, in: Proceeding of seminar on IPM special issue, Indian cotton promotion association, Mumbai. 107110.

Perlak, F.J., Deaton, R.W., Armstrong, T.A., Fuchs, R.L., Sims, S.R., Greenplate, J.T and Fischoff, D.A. 1990. Insect resistant cotton plants. Biotechnology. 8: 939943.

Seshamahalakshmi, M., 2007. Impact of $B t$ cotton on the incidence and management of bollworm complex. Ph.D. Thesis. Acharya N. G. Ranga Agricultural University, Rajendranagar, Hyderabad.

Soujanya, L. P., 2009. Effect of Bt toxins (Cry1Ac \& Cry1Ac + Cry2Ab) on the development \& management of bollworm complex with special reference to Pectinophora gossypiella (Saunders) \& S. litura (Fabracius) on cotton. M. Sc Thesis. Acharya N.G Ranga Agricultural University, Hyderabad.

Sparks, A.N., and Norman, J.W. 2002. Effect of leaf age on efficacy of Bollgard II against beet armyworm Spodoptera exigua. Proceedings of the Beltwide Cotton Conferences, National cotton council of America, Nashville, USA.238-240.

Vennila, S., Panchbhai, P.R and Biradar, V.K. 2006. Growth and survival of Helicoverpa armigera (Hubner) and Spodoptera litura (Boisd.) on transgenic Bt cotton. Journal of Cotton Research and Development. 20(1): 131-133.

Zeng, X.H., Zhang, H.Y., Yu, Z.N and Hu, C. 1998. Bioassay method of evaluating toxicity of Bacillus thuringiensis against larvae of Spodoptera exigua. Chinese Journal of Biological Control. 14(4): $172-175$.

\section{How to cite this article:}

Venkatesh, Y.N., N.V.V.S.D. Prasad, K.B. Ramesh, N. Srinivasa and Sai Ram Kumar, D.V. 2017. Growth and Development of Spodopter litura (fab.) on Dual Toxin Transgenic Bt Cotton. Int.J.Curr.Microbiol.App.Sci. 6(10): 2204-2214. doi: https://doi.org/10.20546/ijcmas.2017.610.261 